\title{
Analysis of an ID-based RF Wake-up Solution for IoT Over Cellular Networks
}

\author{
Nour Kouzayha, Hesham ElSawy, Zaher Dawy and Jeffrey G. Andrews
}

\begin{abstract}
This paper studies the performance of an energy efficient ID-based wake-up solution for Internet of Things (IoT) devices over cellular networks. When the IoT device has no data to transmit, it turns its main circuitry off and switches to sleep mode. The transition back to the active mode is only achieved upon receiving a radio frequency ( $\mathrm{RF}$ ) wake-up signal from the device's serving base station (BS) containing the device's ID. The device experiences a successful wake-up event when it receives enough power to activate an added wake-up module that decodes the received wake-up signal and triggers the main circuitry if the ID of the device is detected. Using tools from stochastic geometry, we define a new composite success wake-up probability metric that takes into consideration the joint effect of sufficient power reception for wake-up and sufficient signal to interference and noise ratio (SINR) for ID detection. The obtained results demonstrate the superiority of the proposed ID-based solution in maintaining efficient performance and low power consumption.

Index Terms-Internet of Things, ID-based RF wake-up, power consumption, cellular network, stochastic geometry.
\end{abstract}

\section{INTRODUCTION}

The Internet of Things (IoT) is expected to entail a massive number of sensors, smart physical objects, and devices that require wireless connectivity [1]. However, unlike humanoperated cellular devices that can be easily charged, IoT devices are often deployed in locations that can be remote and not easily reachable and are usually powered by batteries with restricted size and capacity. This increases the interest in power-efficient communication of IoT, both from system design [2], [3], and hardware perspectives [4].

$\mathrm{RF}$ wake-up is one of the key solutions for ultra-low power consumption [5]. The device enters a deep sleep mode to save power whenever it is not performing a useful task and only leaves the sleep mode when it receives an external RF wake-up signal. A new module must be added to capture the wake-up signal and initiate an interrupt trigger to activate the main circuit of the device. An ID detector can be also added for selective wake-up and the solution in that case is "ID-based". If the ID detector is not present, the wakeup solution is "broadcast-based", and all the devices within the range of the transmitted signal wake-up upon detecting enough power which results in many unnecessary wake-ups. The on-demand characteristic of RF wake-up solutions makes them more suitable for the infrequent transmissions of most

N. Kouzayha and Z. Dawy are with the Electrical and Computer Engineering Department, American University of Beirut, Lebanon. Email: nhk16@aub.edu.lb, zd03@aub.edu.lb. H. ElSawy is with King Abdullah University of Science and Technology (KAUST), Saudi Arabia. Email: hesham.elsawy@kaust.edu.sa. J. G. Andrews is with the Wireless Networking and Communication Group, The University of Texas at Austin, Austin TX, USA. Email: jandrews@ece.utexas.edu.
IoT applications than periodic-based solutions which introduce long delays and are highly power inefficient.

In this work, we present an analytical framework that uses tools from stochastic geometry to evaluate the performance of RF wake-up strategies for IoT devices over cellular networks. Stochastic geometry has been widely applied for the systemlevel analysis of cellular networks [6], [7], particularly to characterize the performance of energy harvesting communications [8]-[10] and wireless powered communications [11]-[14]. For instance, using tools from stochastic geometry, the authors in [8] evaluate the probability of uplink transmission defined as the event of harvesting enough energy to transmit. In [13], the authors use stochastic geometry to study outage and average energy harvested in wireless powered communications.

Unlike the previous works, we study the performance of an IoT cellular network, where the devices, which are equipped with a wake-up module and an ID detector, are initially in the sleep state. Upon reception of a wake-up signal with sufficient amount of power containing the ID of the device, the device switches to the active mode. We propose and evaluate a new composite success wake-up probability metric, where a device encounters a successful wake-up if the received power is sufficient to activate the wake-up module and the ID detector, and the received SINR is sufficient for correct ID detection. Since the same BSs and channels are used for power transfer and ID transmission, there is an inherent correlation in the wake-up and the ID detection events and the joint consideration requires careful analysis, which can be considered the main contribution of this work. Our results clearly prove the superiority of the ID-based solution in providing high energy savings and good success wake-up probability.

\section{System Model AND Assumptions}

The network model consists of BSs arranged according to a homogeneous Poisson point process (PPP) $\Phi=\left\{x_{i}, i=\right.$ $1,2, \ldots\} \in \mathbb{R}^{2}$ with density $\lambda$ in the Euclidean plane. The devices are distributed in $\mathbb{R}^{2}$ according to an independent PPP $\Phi_{u}=\left\{y_{i}, i=1,2, \ldots\right\}$ with density $\lambda_{u}$. We assume that all BSs are transmitting with the same power $P_{t}$ in the downlink (DL). Channels are assumed to be symmetric where the power of a signal transmitted by a BS is subject to the standard power loss propagation model with rate $r^{-\alpha}$ where $\alpha>2$ is the pathloss exponent and $r$ is the propagation distance.

In the analysis, we consider the following assumptions: (i) each device connects to its nearest BS, (ii) fading gains across all channels are independent, (iii) all channels are exposed to Rayleigh fading. This means that the channel power gain $h_{x}$ follows an exponential distribution with unit mean, i.e., 


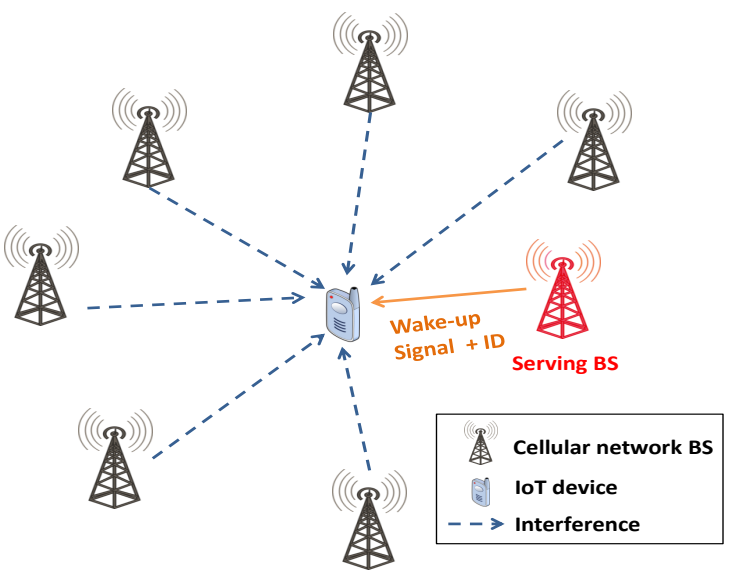

Fig. 1. System model of the ID-based wake-up solution.

$h_{x} \backsim \exp (1)$ and the DL received power at a device located at $y=\left(y_{1}, y_{2}\right)$ from a BS at $x=\left(x_{1}, x_{2}\right)$ is $P_{t} h_{x}\|x-y\|^{-\alpha}$ where $\|$.$\| is the Euclidean norm. Without loss of generality,$ we focus our analysis on a device placed at the origin.

The total received power $P_{r}$ at the device of interest can be expressed as

$$
P_{r}=a \sum_{x \in \Phi} P_{t} h_{x}\|x\|^{-\alpha},
$$

where $a \leq 1$ represents the efficiency of the RF-DC conversion. For the DL, The SINR of a device at the origin can be expressed as

$$
\mathrm{SINR}=\frac{a P_{t} h_{x_{1}}\left\|x_{1}\right\|^{-\alpha}}{\sum_{x \in \Phi \backslash x_{1}} a P_{t} h_{x}\|x\|^{-\alpha}+\sigma^{2}}=\frac{a P_{t} h_{x_{1}}\left\|x_{1}\right\|^{-\alpha}}{I+\sigma^{2}},
$$

where $x_{1}$ is the location of the nearest (serving) BS, $I$ denote the interference field and $\sigma^{2}$ models thermal noise power.

\section{WAKe-UP PRoBabilities ANALYSIS}

\section{A. ID-based Success Wake-up Probability}

As shown in Fig. 1], successful wake-up requires a minimum amount of power to be received at the device in order to activate the circuitry of the wake-up module and the ID detector. Once activated, the ID detector extracts the ID from the wake-up signal and compare it with the ID of the device. In case matched, it sends an interrupt trigger to switch the main circuit to the active mode and initiate operation. Thus, two conditions are required for successful wake-up:

- $P_{r} \geq T$ : The probability that the received power level $P_{r}$ must exceed a power threshold denoted as $T$ to activate the added wake-up module and the ID detector.

- SINR $\geq \beta$ : The received SINR needs to be greater than a modulation-and-coding specific target $\operatorname{SINR} \beta$ to correctly decode the ID from the received wake-up signal.

Therefore, the ID-based success wake-up probability denoted as $P_{\mathrm{ID}}$ is the joint probability of these two events and can be defined as

$$
P_{\mathrm{ID}}=\mathbb{E}\left[\mathbb{1}\left(P_{r} \geq T\right) \mathbb{1}(\mathrm{SINR} \geq \beta)\right] .
$$

Along with the joint treatment, the complexity of the analysis is evident from the additional two facts: (i) the total power received $P_{r}$ is a power-law shot noise whose probability density function (PDF) cannot be derived in general, and (ii) the fading gains are the same for the received power $P_{r}$ and the DL SINR. To make matters worse, the two events both depend on the point process $\Phi$ modeling the BSs. Thus, the two events that compose the joint wake-up probability are dependent and joint analysis is required. To reduce the complexity of the analysis, we start by conditioning on the PPP of the BSs locations $\Phi$. Consequently, the only remaining dependency is the fading gains and the joint wake-up probability can be expressed as

$$
P_{\mathrm{ID}}=\mathbb{E}_{\Phi}\left[\mathbb{P}\left(P_{r} \geq T, \text { SINR } \geq \beta \mid \Phi\right)\right]
$$

To simplify the analysis, we utilize the dominant BS-based approach used in [15] to approximate the total interference $I$ by the interference from the second nearest BS (strongest interferer) and the average of the interference from the rest of the BSs (conditioned on the locations of the nearest two BSs located at distances $r_{1}=\left\|x_{1}\right\|$ and $r_{2}=\left\|x_{2}\right\|$ from the device at the origin) as follows:

$$
I=a \sum_{x \in \Phi \backslash x_{1}} P_{t} h_{x}\|x\|^{-\alpha} \approx a P_{t} h_{x_{2}} r_{2}^{-\alpha}+\psi\left(r_{2}\right),
$$

where $\psi\left(r_{2}\right)=\mathbb{E}\left[\sum_{x \in \Phi \backslash x_{1}, x_{2}} a P_{t} h_{x}\|x\|^{-\alpha} \mid x_{1}, x_{2}\right]$. The value of $\psi\left(r_{2}\right)$ can be derived as follows:

$$
\begin{aligned}
\psi\left(r_{2}\right) & =\mathbb{E}\left[\sum_{x \in \Phi \backslash x_{1}, x_{2}} a P_{t} h_{x}\|x\|^{-\alpha} \mid x_{1}, x_{2}\right] \\
& \stackrel{(a)}{=} \mathbb{E}\left[\sum_{x \in \Phi \backslash x_{1}, x_{2}} a P_{t}\|x\|^{-\alpha}\right] \\
& \stackrel{(b)}{=} 2 \pi \lambda a P_{t} \int_{r_{2}}^{\infty} \frac{1}{r^{\alpha}} r \mathrm{~d} r=\frac{2 \pi \lambda a P_{t}}{\alpha-2} r_{2}^{2-\alpha},
\end{aligned}
$$

where (a) comes from the assumption that all $h_{x}$ are independent exponentially distributed variables with unit mean, and (b) is a direct application of Campbell's theorem for sum over PPP [16]. After some mathematical derivations, the ID-based success wake-up probability can be expressed as in Theorem 1 . Theorem 1 (ID-based success wake-up probability). In an IoT cellular network, assuming that the BSs constitute a PPP with density $\lambda$ and each $B S$ is transmitting with power $P_{t}$, the ID-based success wake-up probability of a generic IoT device with received power threshold $T$, and $D L$ SINR threshold $\beta$ can be derived as

$$
\begin{aligned}
& P_{\mathrm{ID}}=\int_{0}^{\mathcal{Z}_{2}} \int_{0}^{r_{2}} f_{R_{1}, R_{2}}\left(r_{1}, r_{2}\right) e^{-r_{1}^{\alpha} \mathcal{G}} \frac{1}{1+\beta \frac{r_{1}^{\alpha}}{r_{2}^{\alpha}}} \mathrm{d} r_{1} \mathrm{~d} r_{2} \\
& +\int_{\mathcal{Z}_{2}}^{\infty} \int_{0}^{r_{2}} f_{R_{1}, R_{2}}\left(r_{1}, r_{2}\right) e^{-r_{1}^{\alpha} \mathcal{F}} \frac{1-e^{-r_{2}^{\alpha}\left(1-\frac{r_{1}^{\alpha}}{r_{2}^{\alpha}}\right) \mathcal{H}}}{1-\frac{r_{\alpha}^{\alpha}}{r_{2}^{\alpha}}} \mathrm{d} r_{1} \mathrm{~d} r_{2} \\
& +\int_{\mathcal{Z}_{2}}^{\infty} \int_{0}^{r_{2}} f_{R_{1}, R_{2}}\left(r_{1}, r_{2}\right) e^{-r_{1}^{\alpha} \mathcal{G}} \frac{e^{-r_{1}^{\alpha}\left(1+\beta_{\frac{1}{\alpha}}^{r_{2}^{\alpha}}\right) \mathcal{H}}}{1+\beta \frac{r_{\alpha}^{\alpha}}{r_{2}^{\alpha}}} \mathrm{d} r_{1} \mathrm{~d} r_{2},
\end{aligned}
$$


where $\mathcal{Z}_{2}=\left[\frac{2 \pi \lambda}{(\alpha-2)} \frac{a P_{t}(1+\beta)}{T-\beta \sigma^{2}}\right]^{\frac{1}{\alpha-2}}, \mathcal{F}=\frac{T}{a P_{t}}-\frac{2 \pi \lambda}{\alpha-2} r_{2}^{2-\alpha}$, $\mathcal{G}=\frac{\beta \sigma^{2}}{a P_{t}}+\frac{2 \pi \lambda \beta}{\alpha-2} r_{2}^{2-\alpha}, \mathcal{H}=\frac{T-\beta \sigma^{2}}{a P_{t}(1+\beta)}-\frac{2 \pi \lambda}{\alpha-2} r_{2}^{2-\alpha}$, and $f_{R_{1}, R_{2}}\left(r_{1}, r_{2}\right)=(2 \pi \lambda)^{2} r_{1} r_{2} e^{-\lambda \pi r_{2}^{2}}$.

Corollary 1. The ID-based success wake-up probability for the case of $\alpha=4$ can be expressed as

$$
\begin{aligned}
P_{\mathrm{ID}} & =(\pi \lambda)^{2} \int_{0}^{\mathcal{Z}_{2}^{2}} e^{-\lambda \pi v} \int_{0}^{v} \frac{e^{-\beta\left(\frac{\sigma^{2}}{a P_{t}}+\frac{\pi \lambda}{v}\right) u^{2}}}{1+\beta \frac{u^{2}}{v^{2}}} \mathrm{~d} u \mathrm{~d} v \\
& +(\pi \lambda)^{2} \int_{\mathcal{Z}_{2}^{2}}^{\infty} e^{-\left(\frac{T-\beta \sigma^{2}}{a P_{t}(1+\beta)}\right) v^{2}} \int_{0}^{v} \frac{e^{-\frac{\beta}{1+\beta}\left(\frac{T+\sigma^{2}}{a P_{t}}\right) u^{2}}}{1-\frac{u^{2}}{v^{2}}} \mathrm{~d} u \mathrm{~d} v \\
& +(\pi \lambda)^{2} \int_{\mathcal{Z}_{2}^{2}}^{\infty} e^{-\left(\frac{T-\beta \sigma^{2}}{a P_{t}(1+\beta)}\right) v^{2}} \int_{0}^{v} \frac{e^{-\frac{\beta}{1+\beta}\left(\frac{T+\sigma^{2}}{a P_{t}}\right) u^{2}}}{1+\beta \frac{u^{2}}{v^{2}}} \mathrm{~d} u \mathrm{~d} v .
\end{aligned}
$$

Proof: See Appendix A

\section{B. Broadcast-based Success Wake-up Probability}

In a broadcast-based wake-up solution, an IoT device wakes-up successfully when it receives a power $P_{r}$ greater then $T$ when the serving BS is transmitting the wake-up signal without any constraint on the DL SINR. The probability of having $P_{r} \geq T$ is denoted as the broadcast-based success wake-up probability and is given by the following Lemma.

Lemma 1 (Broadcast-based success wake-up probability). The success wake-up probability of an IoT device applying the broadcast-based wake-up solution with power threshold T can be derived as

$$
\begin{aligned}
P_{\text {bro }} & =1-e^{-\lambda \pi \mathcal{Z}_{1}^{2}}-\lambda \pi \mathcal{Z}_{1}^{2} e^{-\lambda \pi \mathcal{Z}_{1}^{2}}+ \\
& \int_{\mathcal{Z}_{1}}^{\infty} \int_{0}^{r_{2}} f_{R_{1}, R_{2}}\left(r_{1}, r_{2}\right) \frac{r_{2}^{\alpha} e^{-r_{1}^{\alpha} \mathcal{F}}-r_{1}^{\alpha} e^{-r_{2}^{\alpha} \mathcal{F}}}{r_{2}^{\alpha}-r_{1}^{\alpha}} \mathrm{d} r_{1} \mathrm{~d} r_{2},
\end{aligned}
$$

where $\mathcal{Z}_{1}=\left[\frac{2 \pi \lambda}{\alpha-2} \frac{a P_{t}}{T}\right]^{\frac{1}{\alpha-2}}, \mathcal{F}=\frac{T}{a P_{t}}-\frac{2 \pi \lambda}{\alpha-2} r_{2}^{2-\alpha}$ and $f_{R_{1}, R_{2}}\left(r_{1}, r_{2}\right)=(2 \pi \lambda)^{2} r_{1} r_{2} e^{-\lambda \pi r_{2}^{2}}$.

Proof: See Appendix B

\section{False Wake-up Probability}

An IoT device implementing the broadcast-based solution wakes-up whenever the received power level exceed $T$ no matter a wake-up signal is designated to it or not. Hence, the broadcast-based solution is vulnerable to frequent false wake-ups. The ID-based solution overcomes this problem by matching the ID of the wake-up signal to the device's ID. Hence, the false wake-up event only activates the wake-up module and the ID detector, which will prevent the unnecessary power of activating the main device's circuitry. The probability of false wake-up is the probability that the received power at the desired device exceeds the threshold $T$ given that the serving BS is not sending a wake-up signal. This is exactly the probability that the received interference exceeds $T$ at the device of interest which can be expressed as in Lemma 2.
Lemma 2 (False wake-up probability). The false wake-up probability of a generic IoT device with power threshold $T$ can be expressed as

$$
P_{\text {false }}=1-e^{-\lambda \pi \mathcal{Z}_{1}^{2}}-\lambda \pi \mathcal{Z}_{1}^{2} e^{-\lambda \pi \mathcal{Z}_{1}^{2}}+\int_{\mathcal{Z}_{1}}^{\infty} f_{R_{2}}\left(r_{2}\right) e^{-r_{2}^{\alpha} \mathcal{F}} \mathrm{d} r_{2}
$$

where $\mathcal{Z}_{1}$ and $\mathcal{F}$ are defined in Lemma 1 and $f_{R_{2}}\left(r_{2}\right)=$ $2(\pi \lambda)^{2} r_{2}^{3} e^{-\pi \lambda r_{2}^{2}}$ is the distribution of the distance to the second nearest BS from the device of interest.

\section{Proof: See Appendix C}

\section{EnERgy CONSUMPTION ANALysis}

In this section, we evaluate the energy savings of the IDbased wake-up solution compared to broadcast and periodicbased solutions. A device employing the periodic-based technique is internally programmed to wake-up at specific intervals and does not require the integration of an extra wake-up module. In order to quantify the consumed energy, we consider a time-slotted system with slot duration $\Delta t$. The time slot is partitioned into two sub-slots: active sub-slot $\left(T_{\mathrm{ON}}\right)$ and sleep sub-slot $\left(T_{\text {sleep }}\right)$. The percentage of time in which the device is active is denoted as $D_{\mathrm{ON}}$. The added wake-up module and ID detector will add extra power consumption to the device in both active and sleep modes. The overall power consumptions of the periodic-based, broadcast-based and ID-based solutions are presented in Table 1 for the success wake-up, false wake-up and sleep modes.

In order to have a successful wake-up, ID-based and broadcast-based solutions require the reception of a wake-up signal during the active sub-slot $T_{\mathrm{ON}}$. However, because of statistical channel effects and decoding errors, the device may fail to wake-up during $T_{\mathrm{ON}}$. As presented in Section III an ID-based wake-up device has a probability $P_{\text {ID }}$ to wake-up successfully. Similarly, a broadcast-based wake-up device has a success wake-up probability $P_{\text {bro }}$ less than 1 . In addition, the device may experience false wake-up events with probability $P_{\text {false }}$ when sufficient amount of power is received during $T_{\text {sleep }}$. Therefore, the total energy consumptions $E_{\text {per }}, E_{\text {bro }}$ and $E_{\mathrm{ID}}$ of periodic-based, broadcast-based and ID-based solutions during the slot duration $\Delta t$ can be respectively estimated as

$$
\begin{aligned}
E_{\mathrm{per}} & =P_{\mathrm{tot}} \Delta t\left[p D_{\mathrm{ON}}+(1-p)\left(1-D_{\mathrm{ON}}\right)\right] \\
E_{\mathrm{bro}} & =(1+y) P_{\mathrm{tot}} \Delta t\left[p P_{\mathrm{bro}} D_{\mathrm{ON}}+(1-p)\left(1-P_{\mathrm{bro}}\right) D_{\mathrm{ON}}\right. \\
& \left.+p P_{\mathrm{false}}\left(1-D_{\mathrm{ON}}\right)+(1-p)\left(1-P_{\mathrm{false}}\right)\left(1-D_{\mathrm{ON}}\right)\right] \\
E_{\mathrm{ID}} & =(1+x+y) P_{\mathrm{tot}} \Delta t\left[p P_{\mathrm{ID}} D_{\mathrm{ON}}\right. \\
& \left.+(1-p)\left(1-P_{\mathrm{ID}}\right) D_{\mathrm{ON}}+(1-p)\left(1-D_{\mathrm{ON}}\right)\right]
\end{aligned}
$$

where $P_{\mathrm{ID}}, P_{\mathrm{bro}}$ and $P_{\text {false }}$ are given in (7), (9) and (10) respectively.

\section{Numerical Results AND Discussions}

In this section, we compare our analytical results to simulation results for all the derived performance metrics. The scenario under consideration is composed of a set of BSs and 
TABLE I

POWER CONSUMPTION FOR DIFFERENT WAKE-UP SOLUTIONS ( $P_{\text {tot }}$ : TOTAL POWER CONSUMPTION, $p$ : ACTIVE POWER PERCENTAGE FOR THE PERIODIC SOLUTION, $y$ : PERCENTAGE OF POWER OF THE WAKE-UP MODULE, $x$ : PERCENTAGE OF POWER CONSUMED FOR ID DETECTION).

\begin{tabular}{|c|c|c|c|}
\hline Solution & Success wake-up & False wake-up & Sleep \\
\hline Periodic-based & $p P_{\text {tot }}$ & - & $(1-p) P_{\text {tot }}$ \\
\hline Broadcast-based & $(1+y) p P_{\text {tot }}$ & $(1+y) p P_{\text {tot }}$ & $(1+y)(1-p) P_{\text {tot }}$ \\
\hline ID-based & $(1+x+y) p P_{\text {tot }}$ & $(1+x+y)(1-p) P_{\text {tot }}$ & $(1+x+y)(1-p) P_{\text {tot }}$ \\
\hline
\end{tabular}

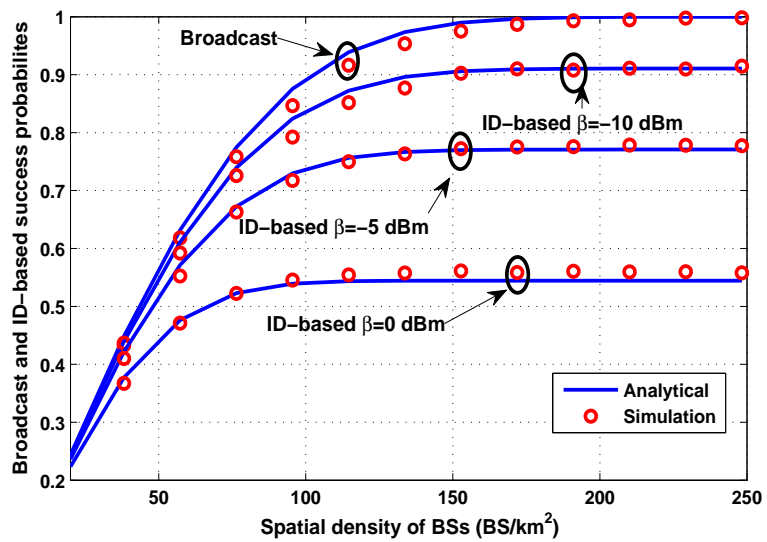

Fig. 2. Broadcast and ID-based success wake-up probabilities as function of the spatial density of BSs $\lambda$ for different values of the SINR threshold $\beta$.

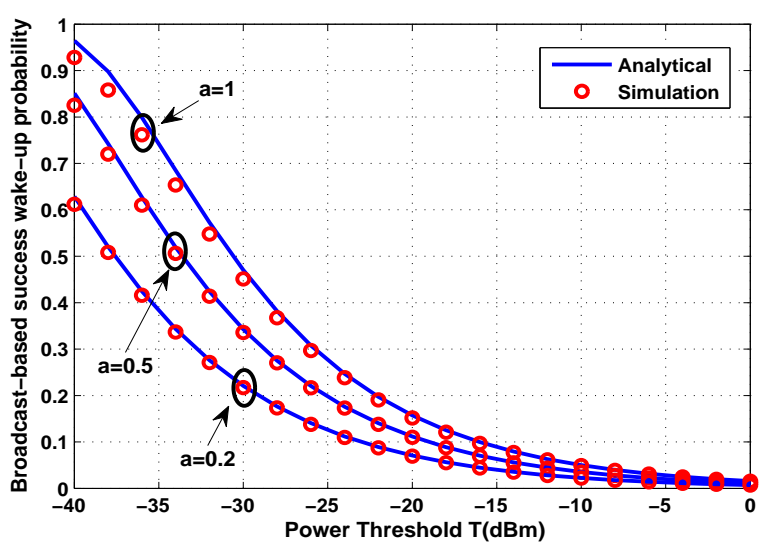

Fig. 3. Broadcast-based success wake-up probability as function of the power threshold $T$ for different values of the RF-DC conversion efficiency factor $a$.

a set of devices distributed following two independent PPPs over the network. Unless otherwise stated, the transmit power of BSs is assumed to be $P_{t}=53 \mathrm{dBm}$ while the thermal noise is $-50 \mathrm{dBm}$ and the wake-up threshold $T$ is $-20 \mathrm{dBm}$. We consider different values for the SINR threshold $\beta$, the pathloss exponent $\alpha$, and the RF-DC conversion factor $a$.

The effect of varying the spatial density of BSs on the IDbased and the broadcast-based success wake-up probabilities is illustrated in Fig. 2 It is easy to see that increasing $\lambda$ increases these probabilities because it reduces the distance $r_{1}$ between the device and its serving BS, which increases the received power, making it easier to satisfy the wake-up threshold condition. This happens up to some point after which deploying more BSs does not affect the overall performance where $P_{\mathrm{ID}}$ and $P_{\mathrm{bro}}$ become almost constant. This behavior can be explained as follows: at high density, the interference power level satisfies the wake-up condition $\mathbb{P}\left(P_{r} \geq T\right)$ literally. Furthermore, the SINR coverage probability is independent of

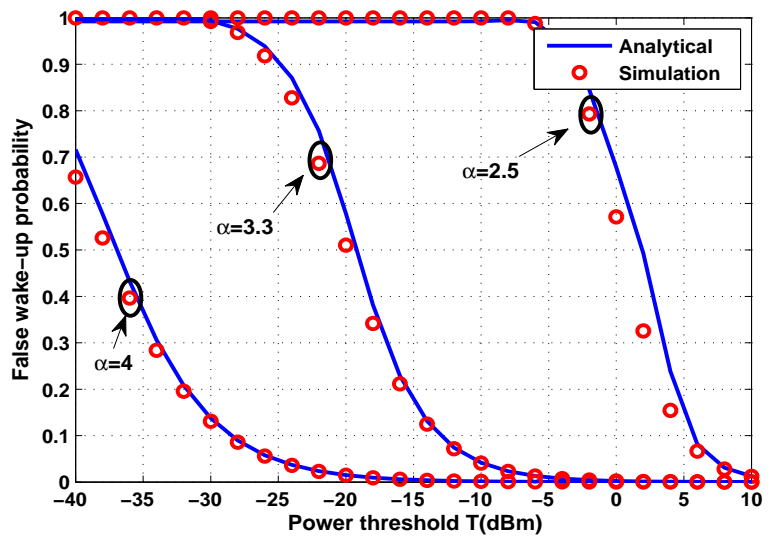

Fig. 4. False wake-up probability as function of the power threshold $T$ for different values of the path loss exponent $\alpha$.

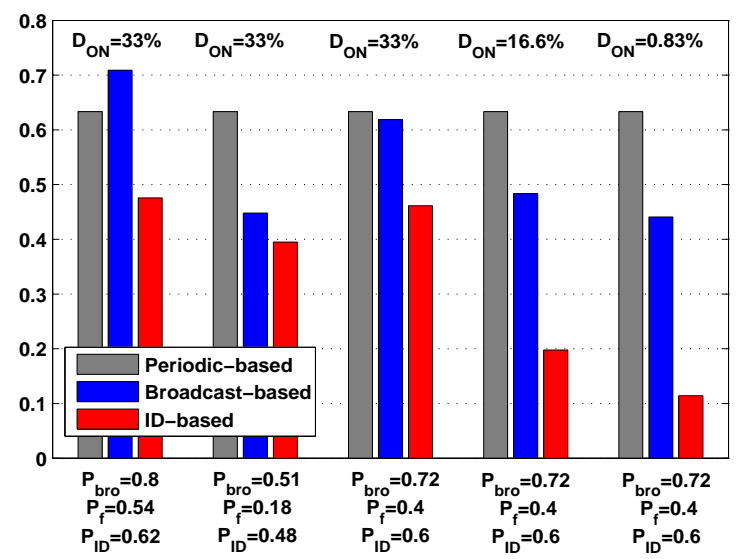

Fig. 5. Energy consumption of periodic, broadcast and ID-based solutions for different values of $D_{\mathrm{ON}}$ and the success and false wake-up probabilities.

$\lambda$ and the total success wake-up probability approaches a constant level for both ID-based and broadcast-based solutions. As shown in Fig. 2 the success wake-up probability derived in Theorem 1 provides a remarkably tight approximation of the true success wake-up probability obtained from simulations. The result for the broadcast-based success wake-up probability presented in Lemma 1 is further validated in Fig. 3 for different values of the RF-DC efficiency conversion factor $a$.

Fig. 4 illustrates the effect of increasing the pathloss exponent $\alpha$ on the false wake-up probability. This figure validates our analytical model for the false wake-up probability through the good match between the analytical results and those obtained from Monte-Carlo simulations for different values of $\alpha$. We note that the false wake-up probability experiences high levels for some network parameters. This is due to the correlation between success and false probabilities. In a broadcast-based wake-up scenario, the high false wake-up rate causes high power consumption levels because the device is 
not able to differentiate between false and true wake-up events and the main circuit is activated at every received power level that exceeds the wake-up threshold $T$. However, in an IDbased scenario, the added ID detector can prevent such false wake-up events by checking the ID included in the received signal, and only interrupting the device in case of matched IDs, therefore preventing unnecessary power loss.

Fig. 5 presents the energy consumption results of the IDbased wake-up solution compared to broadcast and periodicbased solutions based on the analysis in Section IV] The active time percentage $D_{\mathrm{ON}}$ is fixed to $33 \%$ for the periodic solution, and it has several values $(33 \%, 16.6 \%$ and $0.83 \%)$ for the broadcast and ID-based solutions corresponding to different IoT use-cases. Fig. 5 shows that broadcast and ID-based solutions can achieve significant energy savings over periodicbased solutions. The energy reduction becomes higher for IoT use-cases with lower active time percentages. This comes at the cost of less reliability since these solutions have a probability less than one to have successful wake-up. However, given the foreseen sporadic traffic patterns in IoT, and because of their on-demand characteristic, these solutions can save significant delays compared to the periodic case, which has to wait the next on period to wake-up. On the other hand, the broadcast-based solution achieves higher success wakeup probability than the ID-based solution (Fig. 2). However, the false wake-up problem is significant in broadcast solutions and the overall power consumption is much more higher than ID-based solutions. In addition, when the wake-up involves uplink transmissions, broadcast solutions result in high intracell interference which can degrade the performance.

\section{CONCLUSION}

In this work, we studied the performance of an ID-based RF wake-up solution for IoT over cellular. To wake-up successfully, a device must receive a wake-up signal with sufficient amount of power, and must correctly decode the ID it contains. We defined a new composite performance metric that considers both the effect of receiving sufficient power to wake-up and achieving sufficient SINR for ID detection. Since the same set of BSs and the same channels are considered for power transfer and ID transmission, the analysis of the proposed metric required precise joint handling, which can be considered the main contribution of this work. A key design outcome is that, with proper network parameters tuning, the ID-based solution can maintain good performance while reducing significantly the energy consumption compared to the broadcast solution, highly affected with false wake-ups. Many future extensions can be applied to this work. For instance, we can study the performance of a joint uplink/downlink scenario, in which the device transmits uplink data after wake-up. Another extension is to assume that the BS resends the wake-up signal in case of failure, which will add further complexity to the analysis.

\section{APPENDIX}

\section{A. Proof of Theorem 1}

Recalling the necessary conditions for successful wake-up given by (3), the conditioned probability can be derived as follows:

$$
\begin{aligned}
P_{\mathrm{ID}}^{\text {cond }} & =\mathbb{P}\left(P_{r} \geq T, \text { SINR } \geq \beta \mid r_{1}, r_{2}\right) \\
& =\mathbb{P}\left(a P_{t} h_{x_{1}} r_{1}^{-\alpha}+\sum_{x \in \Phi \backslash r_{1}} a P_{t} h_{x} x^{-\alpha} \geq T,\right. \\
& \left.\frac{a P_{t} h_{x_{1}} r_{1}^{-\alpha}}{\sum_{x \in \Phi \backslash r_{1}} a P_{t} h_{x} x^{-\alpha}+\sigma^{2}} \geq \beta\right) \\
& \stackrel{(a)}{=} \mathbb{E}_{h_{x_{2}}}\left[\mathbb { P } \left(h_{x_{1}} \geq r_{1}^{\alpha}\left(\frac{T}{a P_{t}}-\frac{2 \pi \lambda}{\alpha-2} r_{2}^{2-\alpha}-r_{2}^{-\alpha} h_{x_{2}}\right),\right.\right. \\
& \left.\left.h_{x_{1}} \geq r_{1}^{\alpha}\left(\frac{\beta \sigma^{2}}{a P_{t}}+\frac{2 \pi \lambda \beta}{\alpha-2} r_{2}^{2-\alpha}+\beta r_{2}^{-\alpha} h_{x_{2}}\right) \mid h_{x_{2}}\right)\right] \\
& \stackrel{(b)}{=} \mathbb{E}_{h_{x_{2}}}\left[\mathbb { P } \left(h_{x_{1}} \geq \max \left\{r_{1}^{\alpha} \mathcal{F}-\frac{r_{1}^{\alpha}}{r_{2}^{\alpha}} h_{x_{2}},\right.\right.\right. \\
& \left.\left.\left.r_{1}^{\alpha} \mathcal{G}+\beta \frac{r_{1}^{\alpha}}{r_{2}^{\alpha}} h_{x_{2}}\right\} \mid h_{x_{2}}\right)\right],
\end{aligned}
$$

where $\mathcal{F}=\frac{T}{a P_{t}}-\frac{2 \pi \lambda}{\alpha-2} r_{2}^{2-\alpha}$ and $\mathcal{G}=\frac{\beta \sigma^{2}}{a P_{t}}+\frac{2 \pi \lambda \beta}{\alpha-2} r_{2}^{2-\alpha}$. Step (a) follows from the approximation presented in (6). Step (b) is obtained by conditioning over the fading gain $h_{x_{2}}$ of the second nearest BS.

We denote by $P_{\mathrm{ID}}^{h_{x_{2}}}$ the conditioned probability over $h_{x_{2}}$. $P_{\text {ID }}^{h_{x_{2}}}$ can be derived as

$$
\begin{aligned}
& P_{\mathrm{ID}}^{h_{x_{2}}}=\mathbb{P}\left(h_{x_{1}} \geq \max \left\{\left(\mathcal{A}\left(r_{1}, r_{2}\right), \mathcal{B}\left(r_{1}, r_{2}\right)\right\}\right)\right. \\
& = \begin{cases}e^{-r_{1}^{\alpha} \mathcal{F}+\frac{r_{1}^{\alpha}}{r_{2}^{\alpha}} h_{x_{2}}} & \text { if } \mathcal{A}\left(r_{1}, r_{2}\right)>\mathcal{B}\left(r_{1}, r_{2}\right) \\
e^{-r_{1}^{\alpha} \mathcal{G}-\beta \frac{r_{1}^{\alpha}}{r_{2}} h_{x_{2}}} & \text { if } \mathcal{A}\left(r_{1}, r_{2}\right) \leq \mathcal{B}\left(r_{1}, r_{2}\right)\end{cases} \\
& = \begin{cases}e^{-r_{1}^{\alpha} \mathcal{F}+\frac{r_{1}^{\alpha}}{r_{2}} h_{x_{2}}} & \text { if } h_{x_{2}}<r_{2}^{\alpha} \mathcal{H} \\
e^{-r_{1}^{\alpha} \mathcal{G}-\beta \frac{r_{1}^{\alpha}}{r_{2}} h_{x_{2}}} & \text { if } h_{x_{2}} \geq r_{2}^{\alpha} \mathcal{H},\end{cases}
\end{aligned}
$$

where $\mathcal{A}\left(r_{1}, r_{2}\right)=r_{1}^{\alpha} \mathcal{F}-\frac{r_{1}^{\alpha}}{r_{2}^{\alpha}} h_{x_{2}} ; \mathcal{B}\left(r_{1}, r_{2}\right)=r_{1}^{\alpha} \mathcal{G}+\frac{\beta r_{1}^{\alpha}}{r_{2}^{\alpha}} h_{x_{2}}$, and $\mathcal{H}=\frac{\mathcal{F}+\mathcal{G}}{1+\beta}=\frac{T-\beta \sigma^{2}}{a P_{t}(1+\beta)}-\frac{2 \pi \lambda}{\alpha-2} r_{2}^{2-\alpha}$.

Averaging over $h_{x_{2}}$, the conditioned success wake-up probability can be expressed as

$$
\begin{aligned}
P_{\mathrm{ID}}^{\text {cond }} & =e^{-r_{1}^{\alpha}[\mathcal{F}]^{+}} \frac{1-e^{-r_{2}^{\alpha}\left(1-\frac{r_{1}^{\alpha}}{r_{2}^{\alpha}}\right)[\mathcal{H}]^{+}}}{1-\frac{r_{1}^{\alpha}}{r_{2}^{\alpha}}} \\
& +e^{-r_{1}^{\alpha} \mathcal{G}} \frac{e^{-r_{2}^{\alpha}\left(1+\beta \frac{r_{1}^{\alpha}}{r_{2}^{\alpha}}\right)[\mathcal{H}]^{+}}}{1+\beta \frac{r_{1}^{\alpha}}{r_{2}^{\alpha}}},
\end{aligned}
$$

where $[x]^{+}=\max \{0, x\}$. By putting $\mathcal{Z}_{1}=\left[\frac{2 \pi \lambda}{\alpha-2} \frac{a P_{t}}{T}\right]^{\frac{1}{\alpha-2}}$ and $\mathcal{Z}_{2}=$
$\left[\frac{2 \pi \lambda}{\alpha-2} \frac{a P_{t}(1+\beta)}{T-\beta \sigma^{2}}\right]^{\frac{1}{\alpha-2}}$, The terms $[\mathcal{F}]^{+}$and $[\mathcal{H}]^{+}$can be
expressed as

$$
[\mathcal{F}]^{+}= \begin{cases}\mathcal{F}=\frac{T}{a P_{t}}-\frac{2 \pi \lambda}{\alpha-2} r_{2}^{2-\alpha}, & \text { if } r_{2} \geq \mathcal{Z}_{1} \\ 0, & \text { if } r_{2}<\mathcal{Z}_{1}\end{cases}
$$

$$
[\mathcal{H}]^{+}= \begin{cases}\mathcal{H}=\frac{T-\beta \sigma^{2}}{a P_{t}(1+\beta)}-\frac{2 \pi \lambda}{\alpha-2} r_{2}^{2-\alpha}, & \text { if } r_{2} \geq \mathcal{Z}_{2} \\ 0, & \text { if } r_{2}<\mathcal{Z}_{2}\end{cases}
$$


Note that $\mathcal{Z}_{2} \geq \mathcal{Z}_{1}$. Thus, we can define three regions for the distance to the second nearest $\mathrm{BS} r_{2}$. The conditioned success wake-up probability can be finally expressed as

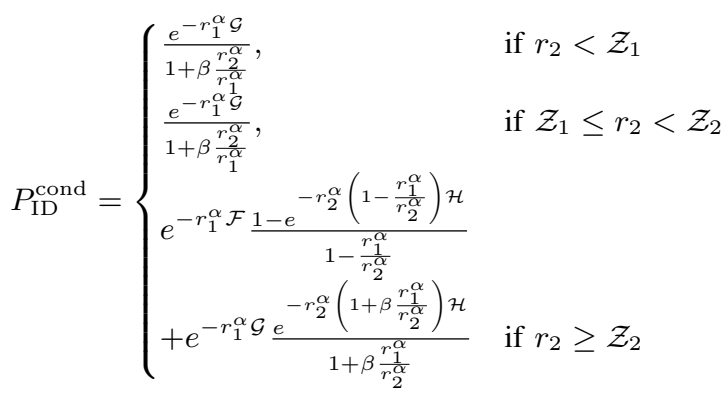

Integrating over $r_{1}$ and $r_{2}$ using the regions defined in (17) and the joint distribution $f_{R_{1}, R_{2}}\left(r_{1}, r_{2}\right)$ defined in [17] leads to the final result in Theorem 1 .

\section{B. Proof of Lemma 1}

To derive the broadcast-based success wake-up probability, we must first obtain the broadcast-based probability conditioned on the PPP of BSs $\Phi$ which reduces to the complementary cumulative density function $(\mathrm{CCDF})$ of $P_{r}$ conditioned on $r_{1}$ and $r_{2}$ and can be expressed as

$$
\begin{aligned}
P_{\mathrm{bro}}^{\text {cond }} & =\mathbb{P}\left(P_{r} \geq T \mid r_{1}, r_{2}\right) \\
& \stackrel{(a)}{=} \mathbb{P}\left(a P_{t} h_{x_{1}} r_{1}^{-\alpha}+a P_{t} h_{x_{2}} r_{2}^{-\alpha}+\frac{2 \pi \lambda a P_{t}}{\alpha-2} r_{2}^{2-\alpha} \geq T\right) \\
& =\mathbb{P}\left(h_{x_{1}} r_{1}^{-\alpha}+h_{x_{2}} r_{2}^{-\alpha} \geq \frac{T}{a P_{t}}-\frac{2 \pi \lambda}{\alpha-2} r_{2}^{2-\alpha}\right) \\
& =\mathbb{P}\left(h_{x_{1}} r_{1}^{-\alpha}+h_{x_{2}} r_{2}^{-\alpha} \geq \mathcal{F}\right) \\
& \stackrel{(b)}{=} \frac{r_{2}^{\alpha} e^{-r_{1}^{\alpha}[\mathcal{F}]^{+}}-r_{1}^{\alpha} e^{-r_{2}^{\alpha}[\mathcal{F}]^{+}}}{r_{2}^{\alpha}-r_{1}^{\alpha}},
\end{aligned}
$$

where (a) follows from approximation presented in (6) and (b) is due to the hypo-exponential distribution of $h_{x_{1}} r_{1}^{-\alpha}+$ $h_{x_{2}} r_{2}^{-\alpha}$. Noting that $[\mathcal{F}]^{+}=0$ and $P_{\text {false }}=1$ when $r_{2} \leq \mathcal{Z}_{1}$ where $\mathcal{Z}_{1}$ is defined in the proof of Theorem 1 and integrating over $r_{1}$ and $r_{2}$ with $f_{R_{1}, R_{2}}\left(r_{1}, r_{2}\right)=(2 \pi \lambda)^{2} r_{1} r_{2} e^{-\lambda \pi r_{2}^{2}}$, the result in Lemma 1 follows.

\section{Proof of Lemma 2}

To derive the false wake-up probability denoted $P_{\text {false }}$, we must obtain the false probability conditioned on the distance $r_{2}$ to the second nearest BS. The conditional probability is exactly the CCDF of the interference field $I$ when no signal is transmitted from its BS and can be expressed as

$$
\begin{aligned}
P_{\text {false }}^{\text {cond }} & =\mathbb{P}\left(I \geq T \mid r_{2}\right) \\
& \stackrel{(a)}{=} \mathbb{P}\left(a P_{t} h_{x_{2}} r_{2}^{-\alpha}+\frac{2 \pi \lambda a P_{t}}{\alpha-2} r_{2}^{2-\alpha} \geq T\right) \\
& =\mathbb{P}\left(h_{x_{2}} r_{2}^{-\alpha} \geq \frac{T}{a P_{t}}-\frac{2 \pi \lambda}{\alpha-2} r_{2}^{2-\alpha}\right) \\
& \stackrel{(b)}{=} \mathbb{P}\left(h_{x_{2}} r_{2}^{-\alpha} \geq \mathcal{F}\right)=e^{-r_{2}^{\alpha}[\mathcal{F}]^{+}},
\end{aligned}
$$

where step (a) follows from the approximation introduced in (6). Step (b) follows from the exponential distribution of $h_{x_{2}}$ with unit mean. Noting that $[\mathcal{F}]^{+}=0$ and $P_{\text {false }}=1$ when $r_{2} \leq \mathcal{Z}_{1}$ and integrating over $r_{2}$ with $f_{R_{2}}\left(r_{2}\right)=$ $2(\pi \lambda)^{2} r_{2}^{3} e^{-\lambda \pi r_{2}^{2}}$ [17] (second nearest distance distribution), the result in Lemma 2 follows.

\section{ACKNOWLEDGMENTS}

This work was made possible by a CNRS-L/AUB PhD Scholarship from the National Council for Scientific Research of the Lebanese Republic (CNRS-L) and the American University of Beirut (AUB). The statements made herein are solely the responsibility of the authors.

\section{REFERENCES}

[1] Z. Dawy, W. Saad, A. Ghosh, J. G. Andrews, and E. Yaacoub, "Toward Massive Machine Type Cellular Communications," IEEE Wireless Communications, vol. 24, pp. 120-128, February 2017.

[2] N. Kouzayha, Z. Dawy, and J. G. Andrews, "Analysis of a Power Efficient Wake-Up Solution for M2M over Cellular Using Stochastic Geometry," in 2016 IEEE Global Communications Conference, December 2016.

[3] G. Zhang, A. Li, K. Yang, L. Zhao, Y. Du, and D. Cheng, "EnergyEfficient Power and Time-Slot Allocation for Cellular-Enabled Machine Type Communications," IEEE Communications Letters, vol. 20, pp. 368-371, February 2016.

[4] N. Mazloum and O. Edfors, "Performance Analysis and Energy Optimization of Wake-Up Receiver Schemes for Wireless Low-Power Applications," IEEE Transactions on Wireless Communications, vol. 13, pp. 7050-7061, December 2014.

[5] N. E. Roberts and D. D. Wentzloff, Ultra-Low Power Wake-Up Radios, pp. 137-162. Springer International Publishing, 2015.

[6] H. ElSawy, A. Sultan-Salem, M. S. Alouini, and M. Z. Win, "Modeling and Analysis of Cellular Networks Using Stochastic Geometry: A Tutorial," IEEE Communications Surveys Tutorials, vol. 19, pp. 167203, Firstquarter 2017.

[7] J. G. Andrews, A. K. Gupta, and H. S. Dhillon, "A Primer on Cellular Network Analysis Using Stochastic Geometry," CoRR, vol. abs/1604.03183, 2016.

[8] A. Sakr and E. Hossain, "Analysis of K-Tier Uplink Cellular Networks With Ambient RF Energy Harvesting," IEEE Journal on Selected Areas in Communications, vol. 33, pp. 2226-2238, October 2015.

[9] I. Flint, X. Lu, N. Privault, D. Niyato, and P. Wang, "Performance Analysis of Ambient RF Energy Harvesting with Repulsive Point Process Modeling,' IEEE Transactions on Wireless Communications, vol. 14, pp. 5402-5416, October 2015.

[10] H. S. Dhillon, Y. Li, P. Nuggehalli, Z. Pi, and J. G. Andrews, "Fundamentals of Heterogeneous Cellular Networks with Energy Harvesting," IEEE Transactions on Wireless Communications, vol. 13, pp. 27822797, May 2014.

[11] K. Huang and V. K. N. Lau, "Enabling Wireless Power Transfer in Cellular Networks: Architecture, Modeling and Deployment," IEEE Transactions on Wireless Communications, vol. 13, pp. 902-912, February 2014.

[12] X. Zhou, R. Zhang, and C. K. Ho, "Wireless Information and Power Transfer: Architecture Design and Rate-Energy Tradeoff," IEEE Transactions on Communications, vol. 61, pp. 4754-4767, November 2013.

[13] I. Krikidis, "Simultaneous Information and Energy Transfer in LargeScale Networks with/without Relaying," IEEE Transactions on Communications, vol. 62, pp. 900-912, March 2014.

[14] M. D. Renzo and W. Lu, "System-Level Analysis/Optimization of Cellular Networks with Simultaneous Wireless Information and Power Transfer: Stochastic Geometry Modeling," IEEE Transactions on Vehicular Technology, vol. 66, pp. 2251-2275, March 2017.

[15] M. A. Kishk and H. S. Dhillon, "Joint Uplink and Downlink Coverage Analysis of Cellular-based RF-powered IoT Network," CoRR, vol. abs/1705.06799, 2017.

[16] S. Chiu, D. Stoyan, W. Kendall, and J. Mecke, Stochastic Geometry and Its Applications. Wiley Series in Probability and Statistics, Wiley, 2013.

[17] D. Moltchanov, "Distance Distributions in Random Networks," Ad Hoc Networks, vol. 10, pp. 1146 - 1166, August 2012. 